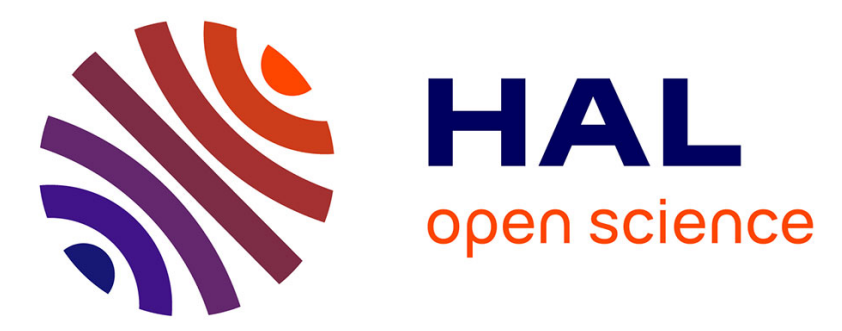

\title{
Accurate Resolution of Time-Dependent and Circuit-Coupled Charge Transport Equations: 1-D Case Applied to 28-nm FD-SOI Devices
}

Victor Malherbe, Gilles Gasiot, Thomas Thery, Jean-Luc Autran, Philippe Roche

\section{- To cite this version:}

Victor Malherbe, Gilles Gasiot, Thomas Thery, Jean-Luc Autran, Philippe Roche. Accurate Resolution of Time-Dependent and Circuit-Coupled Charge Transport Equations: 1-D Case Applied to 28-nm FD-SOI Devices. IEEE Transactions on Nuclear Science, 2018, 65 (1), pp.331-338. 10.1109/TNS.2017.2774960 . hal-02111080

\section{HAL Id: hal-02111080 \\ https://hal-amu.archives-ouvertes.fr/hal-02111080}

Submitted on 1 May 2019

HAL is a multi-disciplinary open access archive for the deposit and dissemination of scientific research documents, whether they are published or not. The documents may come from teaching and research institutions in France or abroad, or from public or private research centers.
L'archive ouverte pluridisciplinaire HAL, est destinée au dépôt et à la diffusion de documents scientifiques de niveau recherche, publiés ou non, émanant des établissements d'enseignement et de recherche français ou étrangers, des laboratoires publics ou privés. 


\title{
Accurate Resolution of Time-Dependent and Circuit-Coupled Charge Transport Equations: 1D Case Applied to $28 \mathrm{~nm}$ FD-SOI Devices
}

\author{
Victor Malherbe* ${ }^{* \dagger}$, Gilles Gasiot*, Thomas Thery*, Jean-Luc Autran ${ }^{\dagger}$, Philippe Roche* \\ ${ }^{*}$ STMicroelectronics, 850 Rue Jean Monnet, F-38926 Crolles Cedex, France. \\ ${ }^{\dagger}$ Aix-Marseille University and CNRS, IM2NP, UMR7334, Marseille, France.
}

\begin{abstract}
We present a 1D drift-diffusion solver for singleevent simulation. Owing to its computational speed and circuitcoupling ability, the module is embedded in our soft error rate simulation platform, enabling projections on logic cells in $28 \mathrm{~nm}$ FD-SOI.
\end{abstract}

\section{INTRODUCTION}

The physics of radiation-induced charge transport and collection is one of the key blocks in the analysis of single event effects on semiconductor devices. Because the constituting equations of electrostatics and carrier motion are tightly coupled, the formulated problem is inherently stiff and best investigated within Technology Computer Aided Design (TCAD) simulations. However, such numerical codes are computationally intensive, typically requiring several hours for a single impact simulation on a 3D structure. Thus TCAD is neither suitable for error rate prediction nor interactive feedback at circuit-design time: both of them require single event models compact enough to execute in seconds, yet physically accurate. Within the fuzzy notion of "physical accuracy", two peculiar features ought to be tackled by such models if they are to be used at analog-circuit ("SPICE") level: time dependence, and circuit coupling.

The Ambipolar Diffusion with Cut-off (ADC) model [1] offered extremely valuable insight into the collection mechanisms, but the analysis was carried out under steady-state conditions only. That same idea of considering purely ambipolar transport in the absence of electric field, followed by collection from the electric field in a depletion region, gave rise to the "diffusion-collection" model [2]: the time-dependent diffusion equation can be solved analytically, however due to the separation between diffusive and drift transport, no true circuit coupling can be implemented. The authors of [3] were among the first to tackle the question of circuit coupling, replacing the (independent) current source by a bias-dependent current-generating network comprised of a capacitor and a (dependent) current source. Circuit-element formulations have also proved to be popular for SOI technology, where bipolar amplification (i.e. additional injection due to the disturbed body potential) is commonly accounted for by injecting the primary radiation-induced current into the base of a bipolar junction transistor [4]. Equivalent-circuit approaches like these can offer great predictive power but only after proper calibration, which is perhaps their main weakness when compared to more physics-based models.

In this paper we develop a single event model based on direct resolution of the semiconductor equations on a onedimensional mesh. The thrust behind this "1D" assumption is twofold: firstly, it greatly alleviates computational cost, allowing to meet the speed requirement for SPICE-level single event models. Secondly, it is physically relevant to advanced technologies such as Fully-Depleted SOI (FD-SOI) or FinFET, which both exhibit active silicon volumes whose dimensionality can be reduced: unlike bulk technology where the transport problem is intrinsically three-dimensional, in $28 \mathrm{~nm}$ FD-SOI the "thickness axis" can actually be collapsed thanks to the ultra-thin $(7 \mathrm{~nm})$ silicon film, and the "width axis" may also be neglected due to the electric field's orientation from drain to source. Last but not least, coupling with an external circuit is enabled thanks to the interactive mode of Mentor Graphics' Eldo circuit solver [5], which makes it possible to run a transient simulation step by step with dynamic access to the circuit parameters.

The paper is organized as follows: we first go through the equations that need to be solved (including how to discretize them properly), and shed some light on practical implementation. We then validate the model against more detailed TCAD simulations, finally to integrate it inside our Monte-Carlo radiation simulator to perform cell-level soft-error studies in $28 \mathrm{~nm}$ FD-SOI.

\section{SOLVER FORMULATION}

\section{A. Physical models}

Guided by Occam's razor, we wish to only include in our solver those physical models that are necessary to account for single event mechanisms, especially in FD-SOI. We thus consider the drift-diffusion transport equations (e.g. instead of more sophisticated hydrodynamic transport):

$$
\begin{gathered}
\nabla \cdot \varepsilon \mathcal{F}=q\left(p-n+N_{d}-N_{a}\right) \\
\frac{\partial n}{\partial t}+R-G-\frac{1}{q} \nabla \cdot \mathcal{J}_{n}=0 \\
\frac{\partial p}{\partial t}+R-G+\frac{1}{q} \nabla \cdot \mathcal{J}_{p}=0 \\
\mathcal{J}_{n}=q \mu_{n} n \mathcal{F}_{n}+q D_{n} \nabla n \\
\mathcal{J}_{p}=q \mu_{p} p \mathcal{F}_{p}-q D_{p} \nabla p
\end{gathered}
$$


(1) is Poisson's equation, where $\varepsilon$ is the dielectric permittivity of silicon, $\mathcal{F}=-\nabla V$ is the electric field obtained from electrostatic potential $V, q$ is the elementary charge, $n$ (resp. $p$ ) is the electron (resp. hole) density, and $N_{d}$ (resp. $N_{a}$ ) is the donor (resp. acceptor) density assumed to be totally ionized. (2) and (3) are the electron and hole continuity equations, where $R$ and $G$ are the recombination and generation terms, and $\mathcal{J}_{n}$ (resp. $\mathcal{J}_{p}$ ) is the electron (resp. hole) current density. Finally (4) and (5) are the drift-diffusion equations for electrons and holes, where $\mu_{n}, D_{n}, \mu_{p}, D_{p}$ are their respective mobilities and diffusivities. The driving fields $\mathcal{F}_{n, p}=-\nabla V_{n, p}=-\nabla\left(V \pm \frac{1}{2} V_{b g n}\right)$ account for the spatial variations of both the electrostatic potential and the energy gap $E_{g, \text { eff }}=E_{g}-q V_{b g n}$, the latter arising because of bandgap narrowing (BGN) at high doping densities. We account for BGN with the del Alamo model [6], and the $\frac{1}{2}$ factors imply equal splitting between the conduction and valence bands. We assume Maxwell-Boltzmann statistics for the carrier concentrations (as opposed to Fermi-Dirac which are more tedious to implement), because our TCAD simulations only showed small differences in terms of radiation currents with or without Fermi statistics (and therefore, the mobilities and diffusivities are related by Einstein's relation: $D=\mu k_{B} T / q$ ). On the other hand, note that overlooking bandgap narrowing leads to completely unrealistic predictions for the bipolar amplification mechanism: when the mass-action law is modified into $(n p)_{e q}=n_{\text {int, eff }}^{2}=n_{\text {int }}^{2} \exp \left(q V_{b g n} / k_{B} T\right)$ where $n_{\text {int }}$ is the intrinsic carrier concentration, then with highly-doped implants the minority concentration is off by an exponential factor, yielding overly pessimistic projections for the parasitic bipolar gain and thus the total collected charge under radiation events. We also model temperature-dependent bandgap and conduction/valence band effective densities of states [7], [8], [9]. Finally, note that we define the electrostatic potential with respect to the intrinsic Fermi level, and therefore at equilibrium we have:

$$
\begin{gathered}
n_{e q}=n_{\text {int }, \text { eff } f} \exp \left(q V / k_{B} T\right) \\
p_{\text {eq }}=n_{\text {int }, \text { eff } f} \exp \left(-q V / k_{B} T\right)
\end{gathered}
$$

Now when outside equilibrium, more specific mechanisms that must be included are:

- Mobility: we model the temperature and doping dependence of $\mu_{n}$ and $\mu_{p}$ via the Arora model [10]. We include velocity saturation (i.e. mobility degradation with the electric field) via the Canali model [11], and take into account mobility degradation with carrier densities (i.e. Carrier-Carrier Scattering or CCS) via the Conwell-Weisskopf model [12]-[13]. Indeed, CCS has been widely recognized as one of the driving mechanisms for radiation-induced carrier transport given the very high injection levels at play [14]. Therefore, it needs to be included in our solver for correct behavior at high Linear Energy Transfers (LET).

- Recombination: we consider Shockley-Read-Hall (SRH) i.e. impurity-assisted recombination depending on the doping level, and Auger i.e. direct band-to-band recom- bination depending on the temperature. Auger recombination becomes especially important at high LET.

- Generation: we use the Van Overstraeten model [15] to account for impact ionization i.e. avalanche multiplication of the carriers under high electric fields. This mechanism is especially important for a proper rendition of bipolar amplification in FD-SOI, which is strongly "avalancheassisted" [16]. Last but not least, the radiation-induced generation of carriers is accounted for with

$$
G_{\text {rad }}(x, t)=N_{\text {rad }}(x) g_{\text {rad }}(t)
$$

where $N_{\text {rad }}(x)$ is the space-dependent deposited density and $g_{\text {rad }}(t)$ is the temporal rate at which deposition occurs.

Finally, we assume electrothermal equilibrium at the contacts at all times. Using $l$ and $r$ subscripts for the left and right sides of the simulation domain, this leads to the following Dirichlet boundary conditions on the primary variables $V, n$, and $p$ :

$$
\begin{gathered}
V\left(x_{l}, t\right)=V_{e q}\left(x_{l}\right)+V_{a, l}(t) \\
n\left(x_{l}, t\right)=n_{e q}\left(x_{l}\right)
\end{gathered}
$$

where $V_{a}$ stands for the voltage applied by the circuit. Equations on the right side and for $p$ were not written for brevity.

\section{B. Spatial and temporal discretization}

In order to yield a system of equations that can actually be solved on a computer, the set of coupled partial differential equations (PDE) above needs to be discretized on a computational grid, both in space and time. The mathematical details of the discretization procedures are given in Appendix A, here we just wish to mention the main steps taken without resorting to formulae:

- The box discretization method [17] is used to discretize (1) (2) and (3), based on the general non-uniform 1D mesh of variable cross section illustrated in Fig. 1. Doing so we can account for the detailed morphology of $28 \mathrm{~nm}$ FD-SOI transistors, whose silicon film thickness is $7 \mathrm{~nm}$ under the gate, but varies near the spacers and goes to $12 \mathrm{~nm}$ in the epitaxy-raised source/drain implants. The electric field $\mathcal{F}$ is easily related to the potential $V$ via a finite-difference formula, but the current densities $\mathcal{J}_{n}$ and $\mathcal{J}_{p}$ cannot be obtained from a naive discretization of (4) and (5): these equations need to be discretized using the Scharfetter-Gummel scheme [18], or else the mesh has to be extremely dense to ensure convergence.

- A backward-Euler approach is used for temporal discretization, for two simple reasons: it is extremely stable (while not the most accurate), and we are anticipating on coupling with an external circuit solver: Eldo's interactive mode allows us to run a transient simulation for arbitrarily small time steps, but it is impossible to "rewind" if we want to subdivide the step further. This prohibits the implementation of a temporal discretization scheme such as the trapezoidal rule, which makes use of "two half time steps" for better accuracy. 


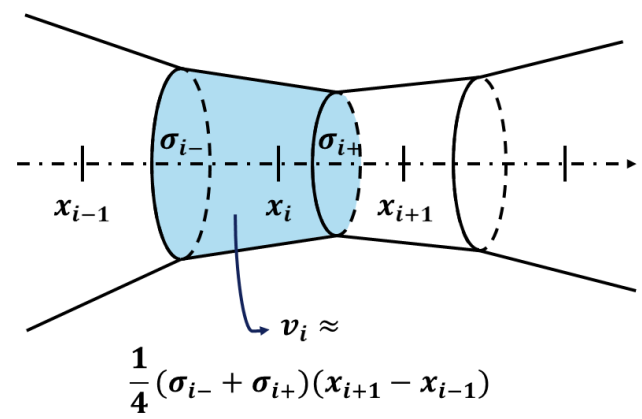

Fig. 1: Notations for 1D mesh of variable thickness along $x$

- Assembling all equations yields a non-linear system for the potentials and carrier densities, from one time step to the other. The system is solved with the Newton-Raphson method, which ultimately amounts to solving a linear system involving the Jacobian matrix of the equation residuals. LU factorization of the Jacobian is the usual procedure for 1D grids, which leads to two triangular systems solved by forward/backward sweep.

- All of the above allows to propagate in time the spacedependent solution, with extrinsic boundary conditions but the circuit response depends itself on the device evolution. Since our circuit solver is a black box, we cannot just append the circuit equations to the device equations, and solve the entire system as one single selfconsistent entity (which is what mixed-mode TCAD often does). Therefore, additional care must be taken with the device-to-circuit communication: a basic implementation of circuit coupling is to substitute the device with a pure current source whose magnitude is calculated by the device solver, then let the circuit solver refresh the terminal voltages, and then compute a new value for the device current, and so forth. Such a coupling method was tested but exhibited mediocre stability, typically requiring time steps below $0.1 \mathrm{ps}$ at all time for convergence. Based on [19], a much better convergence radius was then obtained by replacing the device with an equivalent current source in parallel with an equivalent conductance, as depicted in Fig. 2. Just like the current value, the conductance value is refreshed at each time step by the device solver (with negligible calculation overhead), then handed over to Eldo for circuit simulation. This allows to raise the time step to values close to the picosecond range during the impact - and much higher after - with very limited accuracy loss.

\section{IMPLEMENTATION DETAILS}

Our model is written in $\mathrm{C}++$, in about 5,000 lines of code. During the transient run, communication with the circuit solver is implemented by redirecting the output flux of our program to the input flux of Eldo in interactive mode: using "pipe" system calls, at each time step we are able to communicate the equivalent current and conductance (device-to-circuit link), and retrieve the updated terminal potentials (circuit-to-device

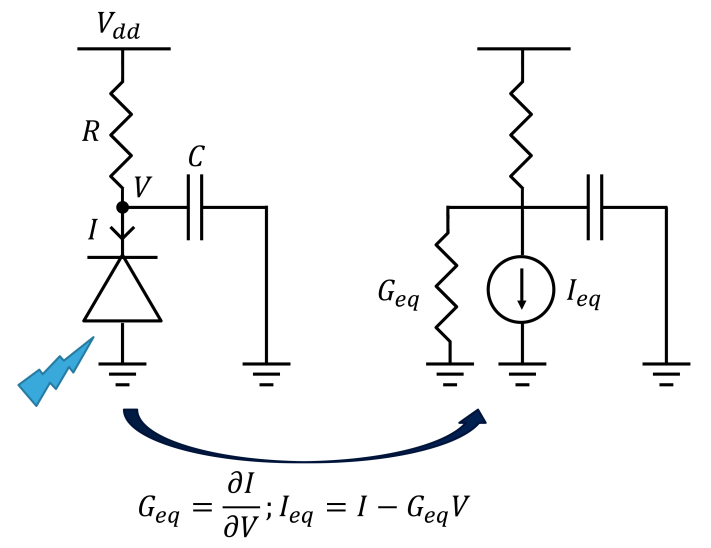

Fig. 2: Circuit coupling with the simulated device's equivalent conductance and current source inside the electrical solver

link). Such an implementation of device-circuit connection was already presented in [20]. Although some of the syntax is necessarily Eldo-specific, this solution can be ported to other electrical solvers as soon as they offer a commandline execution mode allowing arbitrary time increments and dynamic access to the circuit parameters. Alternatively, other works have demonstrated the use of Verilog-A models to perform a similar task [3]: these are really just different technical solutions to tackling the same challenge. Note also that before the transient simulation can be run, the solver first needs to find the equilibrium situation (zero-bias steady state, which solves (1) self-consistently with (6) and (7) starting from an initial guess based on charge neutrality), then perform a quasi-stationary ramp-up (steady state with a bias) so the device is ready to be irradiated.

Now, several optimizations are needed to ensure fast execution:

- First of all, the linear system obtained for the Newton method needs to be built before it can be inverted. Calculating all of the system's protagonists is very lengthy if nothing is done to speed-up the expression evaluations. To tackle this issue, we adopt a pragmatic, hybrid strategy by caching the "clearly re-usable" arrays. For instance, the SRH recombination term is calculated with a fraction $N / D$ on each grid point, so storing the $N$ and $D$ arrays allows for faster evaluation of $\left(N^{\prime} D-N D^{\prime}\right) / D^{2}$ in the Jacobian calculations later on. This requires some hard-coding of the dependence tree in the expression evaluations, to avoid refreshing values in the wrong order. Overall, we observed a near $10 \times$ speed-up when going from a careless evaluation methodology to this hybrid solution. Therefore a complete handling of expression trees - which would be required for optimal lazy reevaluation - was deemed unnecessary.

- Second of all, the system needs to be inverted. For this we make use of Eigen [21], a very complete library for linear algebra. It allows to analyze the sparsity pattern of the Jacobian matrix to accelerate its LU factorization afterwards: the non-zero entries do vary but their indices do not, so the sparsity analysis can be performed once and for all beforehand. We observed a $40 \%$ performance 

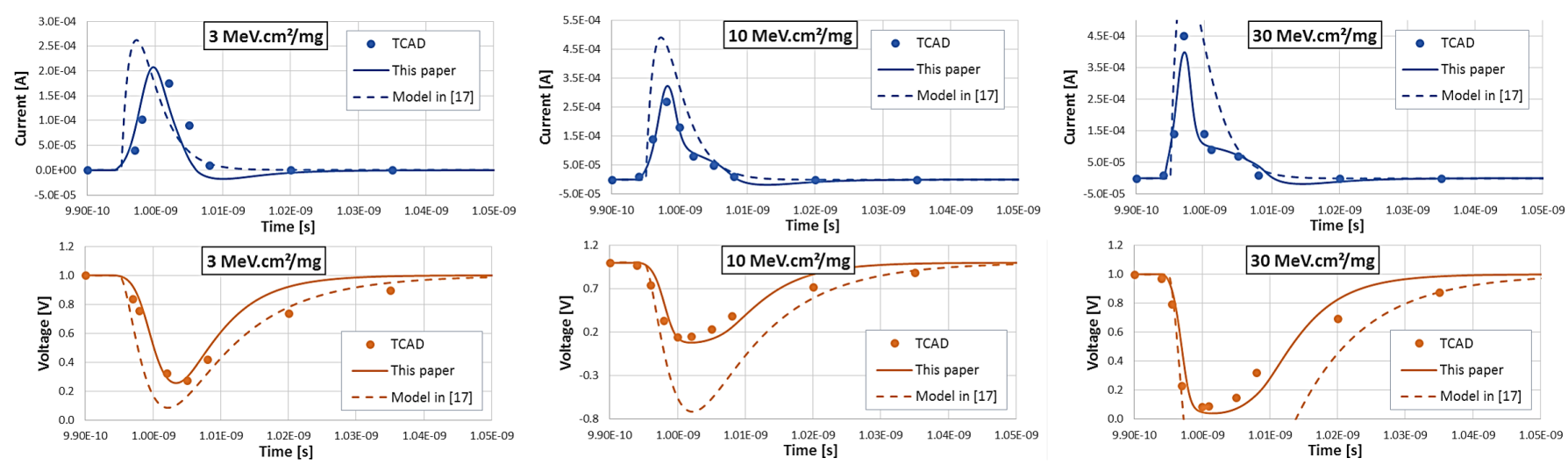

Fig. 3: Comparison between our model and 3D TCAD simulations for various impacts on an NMOS inside an inverter

improvement with this feature - which ironically made the system faster to invert than to build. Furthermore, Eigen enables many compiler optimizations to vectorize the calculations, yielding an additional $3 \times$ speed-up. We also implement some adaptive time step control tailored to our radiation context, with time step relaxation after the impact for instance. The end result of all these optimizations is that an impact takes about 1-3 seconds to run, depending on its toughness: this perfectly meets our speed requirements for Soft-Error Rate (SER) simulation.

\section{Model USAGe IN 28 NM FD-SOI}

\section{A. Validation against $3 D T C A D$}

As a benchmark to validate our model in FD-SOI technology, we compare the results of our 1D solver with more detailed TCAD simulations. The latter are performed by striking a 3D NMOS structure connected in mixed mode to a PMOS and a load capacitance, to simulate the context of an inverter in a CMOS network. Our 1D solver simply takes as input the 1D doping profile of the struck NMOS along its channel axis. A very important note is that in our model, we do not "erase" the NMOS in the circuit simulation, unlike what was shown in Fig. 2 for a toy circuit: we cannot realistically hope for our solver to reproduce the full behavior of a MOSFET since the influence of the gate cannot be natively treated in 1D. Therefore, for the equivalent current and conductance calculations we subtract the steady-state leakage predicted by our solver, and keep the MOSFET in parallel with those circuit elements. In other words, we leave the silicon-calibrated SPICE transistor in charge of the "normaloperation" current, and our solver is only responsible for the radiation-induced current. From a device-level perspective, our simulator is accountable for the motion of excess carriers but not the "background" electrons and holes.

Fig. 3 displays the current and voltage waveforms obtained for impacts at the drain-channel junction of the transistor (where the electric field is highest), for various LET. For comparison we also include results from the behavioral model in [22], where the current waveform was simply a double-exponential pulse, of integral charge given by an LET-dependent bipolar amplification modulated by a position-dependent collection efficiency. As can be seen, the time-dependent effects are well accounted for by our new model, which natively enlarges the current pulse in high injection. On the other hand with the simple model, at high LET the decoupled current source is way off since it does not vanish when the potential drops, thus causing unphysical values for the voltage. This is not an issue for Single-Event Upset (SEU) simulation which is merely interested in whether the voltage does drop or not. But Single-Event Transient (SET) simulations would be quite coarse with the simple model, since the voltage pulse duration is mispredicted.

Mention should be made that the model developed in this paper is not exempt from calibration: the TCAD simulations shown here use hydrodynamic transport equations with more complex mobility modeling (with velocity saturation and interface degradation), beside the models already listed in section II-A. Thus some adjustments are necessary, mainly by playing on the carrier mobilities and lifetimes. Note however that obtaining a good match with TCAD in not an end per se: silicon measurements - e.g. comparison with SEU cross sections on sequential cells - still have to remain the main judge to close the "model calibration" loop.

\section{B. On the importance of circuit coupling}

As a mere sanity check, in Fig. 4 we plotted the waveforms obtained with our model for a $10 \mathrm{MeV} \cdot \mathrm{cm}^{2} / \mathrm{mg}$ impact on a p$\mathrm{n}^{+}$structure with mesh dimensions representative of a bulk-Si junction as depicted in Fig. 2, with $V_{d d}=1.2 \mathrm{~V}, R=10 \mathrm{k} \Omega$, and $C=1 \mathrm{fF}$. As can be seen, our 1D solver perfectly captures the so-called "plateau" effect [23], which arises in modern bulk technologies when the time constants for charge transport and circuit response start overlapping. The $\mathrm{n}^{+}$voltage is clamped at $-V_{b i}=-0.8 \mathrm{~V}$, while the current lingers at the precise value of $\left(V_{d d}+V_{b i}\right) / R=(1.2+0.8) / 10,000=0.2 \mathrm{~mA}$ : if the current was to exceed this value, the $\mathrm{n}^{+}$potential would drop even further and the junction would become truly forward biased, thus it would start driving a massive current in the opposite direction that would actually draw the potential back up very quickly. As a matter of fact, this phenomenon can be observed when intentionally using overly relaxed time steps during the plateau: the forward-bias current restores a 
dramatically high potential on the $\mathrm{n}^{+}$node, which causes spurious oscillations that sometimes stabilize, or sometimes can cause the simulation to diverge.

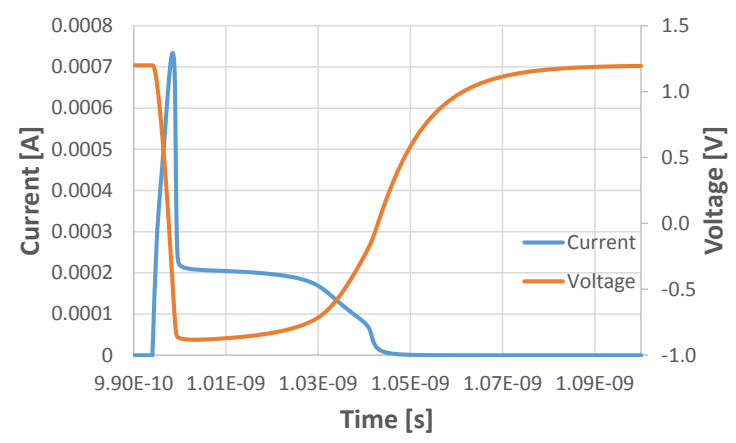

Fig. 4: Sanity check for circuit coupling: plateau effect for a $10 \mathrm{MeV} . \mathrm{cm}^{2} / \mathrm{mg}$ impact on a mesh representative of a bulk-Si $\mathrm{p}-\mathrm{n}^{+}$junction connected like in Fig. 2. The plateau lasts for as long as there are charges to collect that have not recombined, and then the voltage recovers with an $R C$ time constant.

On the other hand as can be seen in Fig. 3, in FD-SOI the plateau effect due to circuit coupling does arise, but in a less pronounced way than in bulk technology. This was expected given the reduced active silicon volumes, providing a much smaller reservoir for continued charge collection over time. More important than the current plateau however is the fact that the $V_{d s}$ voltage of the MOSFET is clamped at 0 , much like it was clamped at $V_{b i}$ for a junction alone. This is of great relevance to properly capture the radiation behavior of devices connected in series. For instance, consider the robust inverter shown in Fig. 5, which uses transistors of identical function in series. Stacking is a well-known method for hardening, and its efficiency has sometimes been explained by the fact that it is highly unlikely for an impact to "turn on" both off-state transistors at the same time, especially in SOI technology. More rigorously, the robustness comes from the fact that since $V_{d s}$ tends to clamp at 0 during an impact, for instance when we strike the NMOS closest to Z, the output cannot drop below $0.5 \mathrm{~V}$ for a long time and therefore, only short-lived SETs could propagate. For the voltage waveform obtained at $30 \mathrm{MeV} . \mathrm{cm}^{2} / \mathrm{mg}$ in Fig. 5, the glitch is likely to be filtered by the next logic stage. For comparison we also plotted the same simulation but without circuit coupling: the current was extracted under the same impact but with a constant $0.5 \mathrm{~V}$ hard bias across the NMOS, and then this frozen current waveform was injected in the circuit simulation to obtain a voltage waveform. As can be seen, in the non-coupled case the potential has no reason not to drop below $0.5 \mathrm{~V}$, and therefore the glitch could very well propagate. This truly establishes the relevance of developing bias-dependent single event models if one is to try and predict the robustness of a given circuit design: without circuit coupling, the simulation simply misses the entire point of hardening by stacking in SOI.

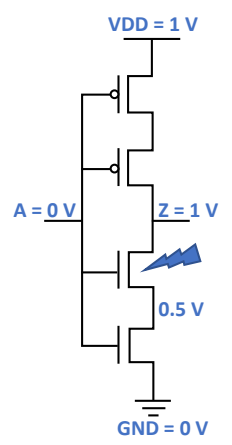

(a)

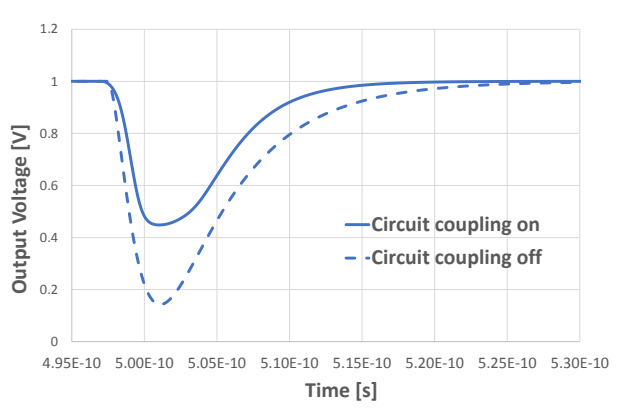

(b)
Fig. 5: In FD-SOI there is no strong current plateau, but the drain-source voltage of the struck FET does clamp at 0 , highlighting the importance of circuit coupling: for a robust inverter that uses identical transistors stacked in series (a), the voltage drop (b) can be overestimated if the radiation current model does not dynamically depend on bias $\left(30 \mathrm{MeV} . \mathrm{cm}^{2} / \mathrm{mg}\right.$ impact).

\section{Cell-level explorations}

In this last section we embed our collection model within our proprietary Monte-Carlo SER simulation platform called TIARA [24]. Briefly speaking, TIARA builds a 3D model of a circuit based on its layout and technological process information. Then using the circuit's netlist, a simplified Layout Versus Schematic (LVS) is run to map the devices and nodes in the schematic to physical locations in the 3D model. The user can then specify an irradiation scenario, and ions are drawn randomly and ray-traced to compute charge deposition inside the structure. Finally, the collection model is called in conjunction with the electrical solver in order to compute the circuit's response to the impacts. Some software upgrades were needed on both sides to enable smooth integration of the new collection model within TIARA. Beside general flow unification tasks to connect the API together, we had to dynamically generate the inputs for the new collector: first, automatic generation of the $1 \mathrm{D}$ meshes based on the impacted transistors, and then conversion of the raytracer's output into a carrier generation term. Note that for the first task, in the case of two transistors sharing a common diffusion area we generate two simulation domains with a shared boundary condition. In Fig. 6 we plotted the results of a virtual heavy-ion irradiation on an $28 \mathrm{~nm}$ FD-SOI SRAM cell: experimental data was obtained at RADEF [25] at room temperature, but high-temperature runs were only carried out for SingleEvent Latchup (SEL) testing. Our simulations show that the saturation cross section is nearly identical at $100^{\circ} \mathrm{C}$, but at low LET it is increased by $60 \%$. This prediction is compatible with our experimental neutron-SER measurements showing a $2 \times$ increase at $125^{\circ} \mathrm{C}$ on similar bit-cells. This can be of importance for space applications, which often feature intense temperature cycles. Note that such an investigation would have been less straightforward with the simple model in [22], which is only calibrated at room temperature: it would have required a TCAD study first, then to introduce an ad-hoc temperature 
dependence in the collection model.

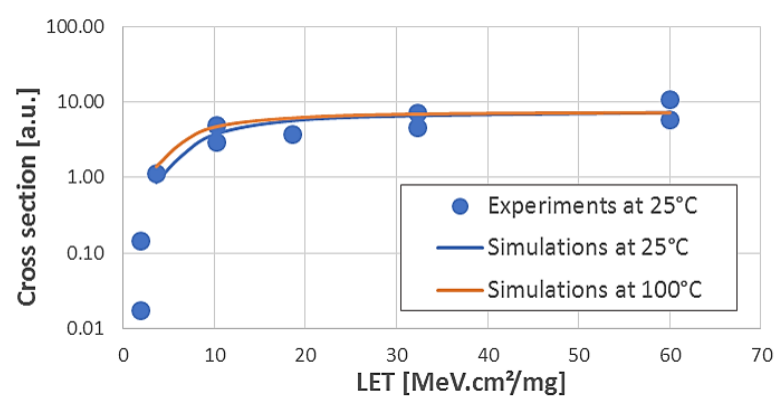

Fig. 6: SRAM cross section simulated at elevated temperature

\section{CONCLUSION}

In this paper we have developed a time-dependent single event model that can be interfaced with an electrical solver. The model is based on direct resolution of the semiconductor equations on a one-dimensional grid, making it computationally efficient. Furthermore this study has shown that the model is very accurate when applied to $28 \mathrm{~nm}$ FD-SOI devices, due to the ultra-thin silicon film in that technology. It has been integrated within our Monte-Carlo radiation simulation platform, highlighting how physics-based models can natively enable advanced sensitivity analyses at cell level. Note that the model could be applied seamlessly to more advanced nodes in FD-SOI - e.g. 22 or $12 \mathrm{~nm}$ - and, with some adaptation, to FinFET technology as well: using simplified 3D diffusion in the bulk beneath the fin and looking at carriers collected at the base of the fin, would allow to derive an effective generation term inside the fin and then, use 1D transport equations as shown in this article. Note that this implicitly assumes that collection inside the fin does not strongly affect transport in the bulk beneath, which seems reasonable since the electric field is mostly confined within the channel.

From a physicist's perspective, compact analytical models are often preferred for the immediate insight they can offer, while brute-force numerical approaches are sometimes regarded as merely "number-crunching" routines making it hard to build one's physical intuition. However in this case we find that, owing to the solver's great speed, having the ability to get an instantaneous answer to any thought experiment - in $1 \mathrm{D}$ - is also extremely valuable and helps us gain a deeper understanding of the underlying mechanisms.

\section{APPENDIX}

Our goal here is to shed more light onto the mathematical details of the discretization procedure that was described succinctly in Section II-B. Suppose we want to compute the solution to our coupled PDE set on discrete grid points $\left\{x_{i}\right\}_{i=0 . . I-1}$ and discrete instants $\left\{t_{j}\right\}_{j=0 . . J-1}$. We will note $f\left(x_{i}, t_{j}\right)=f_{i, j}$ for short. We also scale our units to work on dimensionless quantities: all potentials are divided by the thermal voltage $V_{T}=k_{B} T / q$, all densities (carriers, doping, radiation...) are divided by the intrinsic density $n_{\text {int }}$, and to close the loop, all times are divided by an arbitrary, extrinsic, reference time.

First, the box method [17] is used for spatial discretization; for all equations of the form $\nabla \cdot \mathcal{F}+S=0$ i.e. (1) (2) and (3), the idea is to use Ostrogradky's theorem on a local control volume (the "box" depicted in Fig. 1), yielding:

$$
\sigma_{i+} \mathcal{F}_{i+}-\sigma_{i-} \mathcal{F}_{i-}+v_{i} S_{i}=0
$$

In Poisson's equation for instance, this replaces the usual finite-difference formula for $\Delta V$ with a reweighted Laplacian operator based on the local mesh cross section, natively leading to steeper potential drops in areas where the silicon is thinner. More precisely, if we denote $d x_{i-}^{2}=v_{i}\left(x_{i}-x_{i-1}\right) / \sigma_{i-}$ and $d x_{i+}^{2}=v_{i}\left(x_{i+1}-x_{i}\right) / \sigma_{i+}$, then the Laplacian stencil is expressed as:

$$
\Delta_{i}=\left[\frac{1}{d x_{i-}^{2}},-\left(\frac{1}{d x_{i-}^{2}}+\frac{1}{d x_{i+}^{2}}\right), \frac{1}{d x_{i+}^{2}}\right]
$$

Now the current equations (4) and (5) are more specific, and need to be discretized via the Scharfetter-Gummel scheme [18]. Briefly summarized, since the electron and hole densities have exponential dependence on the potential, when using naive finite-difference formulae the equations are made locally inconsistent unless the mesh is extremely dense. The Scharfetter-Gummel method prevents this issue by anticipating the values of $n$ and $p$ in-between grid points, yielding e.g. for the leftward current densities:

$$
\begin{aligned}
\mathcal{J}_{n, i-} & =\mu_{n, i-}\left[n_{i} B\left(V_{n, i}-V_{n, i-1}\right)-n_{i-1} B\left(V_{n, i-1}-V_{n, i}\right)\right] \\
\mathcal{J}_{p, i-} & =\mu_{p, i-}\left[p_{i-1} B\left(V_{p, i}-V_{n, i-1}\right)-p_{i} B\left(V_{p, i-1}-V_{p, i}\right)\right]
\end{aligned}
$$

where the Bernoulli function:

$$
B(x)=\frac{x}{\mathrm{e}^{x}-1}
$$

performs an optimal transition from a usual finite difference formula (which works well for diffusion-driven transport) to an upwind difference formula (which works well for driftdominated problems) depending on carrier velocity. Note that $B(x)$ needs to be evaluated carefully to avoid overflows at large values of $x$ and imprecision due to the indeterminate form when $x \rightarrow 0$ (in that case we use a Taylor series expansion evaluated to machine precision).

As for temporal discretization, with the backward-Euler method all expressions of the form $\partial g / \partial t+h=0$ are replaced by $\left(g_{j}-g_{j-1}\right) /\left(t_{j}-t_{j-1}\right)+h_{j}=0$. Then when going from $j-1$ to $j$, assembling all discretized equations (with (4) and (5) substituted into (2) and (3)) yields a non-linear system of $3 I$ equations for $3 I$ unknowns - which we shall refrain from writing here in its entirety for the sake of compactness; if we denote our state vector $X$, then it is of the form:

$$
0_{3 I}=f\left(V_{0, j} . . V_{I-1, j}, n_{0, j} . . n_{I-1, j}, p_{0, j} . . p_{I-1, j}\right)=f\left(X_{j}\right)
$$

The root to the equation residuals $f$ is computed via the Newton-Raphson method, which performs successive refinements to the solution by linearizing the system. To go from 
"outer" iteration $k$ to $k+1$ we compute an update candidate $d X_{j}^{k+1}$ as the solution to the linearized system:

$$
0_{3 I}=J_{f} \cdot d X_{j}^{k+1}+f\left(X_{j}^{k}\right)
$$

where $J_{f}$ stands for the Jacobian matrix of $f$. Then to actually refresh the solution we compute

$$
X_{j}^{k+1}=X_{j}^{k}+\lambda^{k+1} d X_{j}^{k+1}
$$

where $\lambda$ is a damping parameter taken "as close as possible to 1 ", based on whether or not the update actually does improve the residuals - typically with geometrical damping for the "inner" iterations. Overall convergence is checked by ensuring the norm of the right-hand side falls below a given value. Optionally, we could also use a criterion on the norm of the solution update, but since the Newton method converges quadratically (doubling the number of exact digits at each step) when initialized close enough to the solution, this could cause to systematically perform one useless iteration just to let the solution stabilize when it is already good enough.

A last remaining mathematical aspect is the calculation of the equivalent conductance for our simulated device. It is defined as $G_{e q}=\partial \mathcal{I} / \partial U$, where the applied voltage difference is $U=V_{l}-V_{r}-\left(V_{0, e q}-V_{I-1, e q}\right)$ and current $\mathcal{I}$ is obtained via the mesh average of the flux of the current densities in order to minimize numerical error:

$$
\mathcal{I}=\frac{1}{I-1} \sum_{i=0}^{I-2}\left(\mathcal{J}_{n, i+}+\mathcal{J}_{p, i+}\right) \cdot \sigma_{i+}
$$

(the displacement current $\mathcal{I}_{d}$ can safely be ignored: even when the voltage drops by $1 \mathrm{~V}$ in $1 \mathrm{ps}$, for our $L \approx 100 \mathrm{~nm}, \sigma \approx$ $10 \times 100 \mathrm{~nm}^{2}$ meshes it only reaches $\mathcal{I}_{d} \approx \varepsilon \sigma / L \cdot \partial U / \partial t \approx$ $1 \mu \mathrm{A})$. Intuitively, the derivative for $G_{e q}$ should not have to be computed by evaluating the solution "at $U+d U$ " - which could double the overall computation time - since the Jacobian matrix $J_{f}$ already contains all the information about how the equation residuals vary around the solution. This is precisely the idea in [19]: we can write

$$
G_{e q}=\frac{\partial \mathcal{I}}{\partial X} \cdot \frac{\partial X}{\partial U}
$$

The first term is evaluated from symbolic differentiation, and to obtain the second term we make use of the fact that

$$
f(X(U), U)=0 \Longrightarrow J_{f} \cdot \frac{\partial X}{\partial U}=-\frac{\partial f}{\partial U}
$$

$\partial f / \partial U$ is a nearly-empty vector since $U$ only appears at the boundary nodes, and because the Jacobian is already LUfactorized, the above system can be solved for $\partial X / \partial U$ at almost no computational overcost.

\section{REFERENCES}

[1] L. D. Edmonds, "A Theoretical Analysis of Steady-State Charge Collection in Simple Diodes Under High-Injection Conditions," IEEE Transactions on Nuclear Science, vol. 57, no. 2, pp. 818-830.

[2] J.-M. Palau, G. Hubert, K. Coulie, B. Sagnes, M.-C. Calvet, and S. Fourtine, "Device simulation study of the SEU sensitivity of SRAMs to internal ion tracks generated by nuclear reactions," IEEE Transactions on Nuclear Science, vol. 48, no. 2, pp. 225-231, Apr 2001.

[3] J. S. Kauppila, A. L. Sternberg, M. L. Alles, A. M. Francis, J. Holmes, O. A. Amusan, and L. W. Massengill, "A Bias-Dependent Single-Event Compact Model Implemented Into BSIM4 and a $90 \mathrm{~nm}$ CMOS Process Design Kit," IEEE Transactions on Nuclear Science, vol. 56, no. 6, pp. 3152-3157.
[4] D. Fulkerson and E. Vogt, "Prediction of SOI single-event effects using a simple physics-based SPICE model," IEEE Transactions on Nuclear Science, vol. 52, no. 6, pp. 2168-2174.

[5] "Eldo Classic - Foundry Certified SPICE Accurate Circuit Simulation." [Online]. Available: https://www.mentor.com/products/ic_nanometer_ design/analog-mixed-signal-verification/eldo/?clp=1

[6] J. del Alamo, S. Swirhun, and R. M. Swanson, "Simultaneous measurement of hole lifetime, hole mobility and bandgap narrowing in heavily doped n-type silicon," in 1985 International Electron Devices Meeting, vol. 31, 1985, pp. 290-293.

[7] W. Bludau, A. Onton, and W. Heinke, "Temperature dependence of the band gap of silicon," Journal of Applied Physics, vol. 45, no. 4, pp. 1846-1848, 1974.

[8] M. A. Green, "Intrinsic concentration, effective densities of states, and effective mass in silicon," Journal of Applied Physics, vol. 67, no. 6, pp. 2944-2954, 1990.

[9] J. E. Lang, F. L. Madarasz, and P. M. Hemenger, "Temperature dependent density of states effective mass in nonparabolic p-type silicon," Journal of Applied Physics, vol. 54, no. 6, pp. 3612-3612, 1983.

[10] N. D. Arora, J. R. Hauser, and D. J. Roulston, "Electron and hole mobilities in silicon as a function of concentration and temperature," IEEE Transactions on Electron Devices, vol. 29, no. 2, pp. 292-295, Feb 1982.

[11] C. Canali, G. Majni, R. Minder, and G. Ottaviani, "Electron and hole drift velocity measurements in silicon and their empirical relation to electric field and temperature," IEEE Transactions on Electron Devices, vol. 22, no. 11, pp. 1045-1047, Nov 1975.

[12] S. C. Choo, "Theory of a forward-biased diffused-junction P-L-N rectifier - Part I: Exact numerical solutions," IEEE Transactions on Electron Devices, vol. 19, no. 8, pp. 954-966, Aug 1972.

[13] N. H. Fletcher, "The High Current Limit for Semiconductor Junction Devices," Proceedings of the IRE, vol. 45, no. 6, pp. 862-872, June 1957.

[14] P. Dodd, "Device simulation of charge collection and single-event upset," IEEE Transactions on Nuclear Science, vol. 43, no. 2, pp. 561-575, Apr 1996.

[15] R. V. Overstraeten and H. D. Man, "Measurement of the ionization rates in diffused silicon p-n junctions," Solid-State Electronics, vol. 13, no. 5, pp. $583-608,1970$.

[16] V. Malherbe, G. Gasiot, D. Soussan, A. Patris, J.-L. Autran, and P. Roche, "Alpha soft error rate of FDSOI $28 \mathrm{~nm}$ SRAMs: Experimental testing and simulation analysis," in 2015 IEEE International Reliability Physics Symposium (IRPS), April 2015, pp. SE.11.1-SE.11.6.

[17] R. E. Bank, D. J. Rose, and W. Fichtner, "Numerical methods for semiconductor device simulation," IEEE Transactions on Electron Devices, vol. 30, no. 9, pp. 1031-1041.

[18] D. L. Scharfetter and H. K. Gummel, "Large-signal analysis of a silicon read diode oscillator," IEEE Transactions on Electron Devices, vol. 16, no. 1 , pp. $64-77$.

[19] K. Mayaram and D. O. Pederson, "Coupling algorithms for mixed-level circuit and device simulation," IEEE Transactions on Computer-Aided Design of Integrated Circuits and Systems, vol. 11, no. 8.

[20] M. Glorieux, J. L. Autran, D. Munteanu, S. Clerc, G. Gasiot, and P. Roche, "Random-Walk Drift-Diffusion Charge-Collection Model For Reverse-Biased Junctions Embedded in Circuits," IEEE Transactions on Nuclear Science, vol. 61, no. 6, pp. 3527-3534, Dec 2014.

[21] G. Guennebaud, B. Jacob et al., "Eigen v3," http://eigen.tuxfamily.org, 2010.

[22] V. Malherbe, G. Gasiot, D. Soussan, J. L. Autran, and P. Roche, "OnOrbit Upset Rate Prediction at Advanced Technology Nodes: a $28 \mathrm{~nm}$ FD-SOI Case Study," IEEE Transactions on Nuclear Science, vol. 64, no. 1, pp. 449-456, Jan 2017.

[23] S. DasGupta, A. F. Witulski, B. L. Bhuva, M. L. Alles, R. A. Reed, O. A. Amusan, J. R. Ahlbin, R. D. Schrimpf, and L. W. Massengill, "Effect of Well and Substrate Potential Modulation on Single Event Pulse Shape in Deep Submicron CMOS," IEEE Transactions on Nuclear Science, vol. 54, no. 6, pp. 2407-2412, Dec 2007.

[24] P. Roche, G. Gasiot, J. Autran, D. Munteanu, R. Reed, and R. Weller, "Application of the TIARA Radiation Transport Tool to Single Event Effects Simulation," IEEE Transactions on Nuclear Science, vol. 61, no. 3, pp. 1498-1500, June 2014.

[25] A. Virtanen, "Radiation effects facility RADEF," in 2002 Proceedings of the Eighth IEEE International On-Line Testing Workshop, 2002, p. 188 . 